\title{
How dielectric screening in two-dimensional crystals affects the convergence of excited-state calculations: Monolayer MoS2
}

\author{
Hüser, Falco; Olsen, Thomas; Thygesen, Kristian Sommer
}

Published in:

Physical Review B Condensed Matter

Link to article, DOI:

10.1103/PhysRevB.88.245309

Publication date:

2013

Document Version

Publisher's PDF, also known as Version of record

Link back to DTU Orbit

Citation (APA):

Hüser, F., Olsen, T., \& Thygesen, K. S. (2013). How dielectric screening in two-dimensional crystals affects the convergence of excited-state calculations: Monolayer $\mathrm{MoS}_{2}$. Physical Review B Condensed Matter, 88(24), [245309]. https://doi.org/10.1103/PhysRevB.88.245309

\section{General rights}

Copyright and moral rights for the publications made accessible in the public portal are retained by the authors and/or other copyright owners and it is a condition of accessing publications that users recognise and abide by the legal requirements associated with these rights.

- Users may download and print one copy of any publication from the public portal for the purpose of private study or research.

- You may not further distribute the material or use it for any profit-making activity or commercial gain

- You may freely distribute the URL identifying the publication in the public portal 


\title{
How dielectric screening in two-dimensional crystals affects the convergence of excited-state calculations: Monolayer $\mathrm{MoS}_{2}$
}

\author{
Falco Hüser, ${ }^{1, *}$ Thomas Olsen, ${ }^{1}$ and Kristian S. Thygesen ${ }^{1,2, \dagger}$ \\ ${ }^{1}$ Center for Atomic-scale Materials Design (CAMD), Department of Physics, Technical University of Denmark, 2800 Kgs. Lyngby, Denmark \\ ${ }^{2}$ Center for Nanostructured Graphene (CNG), Technical University of Denmark, 2800 Kgs. Lyngby, Denmark
}

(Received 22 July 2013; revised manuscript received 29 October 2013; published 26 December 2013)

\begin{abstract}
We present first-principles many-body calculations of the dielectric constant, quasiparticle band structure, and optical absorption spectrum of monolayer $\mathrm{MoS}_{2}$ using a supercell approach. As the separation between the periodically repeated layers is increased, the dielectric function of the layer develops a strong $q$ dependence around $q=0$. This implies that denser $k$-point grids are required to converge the band gap and exciton binding energies when large supercells are used. In the limit of infinite layer separation, here obtained using a truncated Coulomb interaction, a $45 \times 45 k$-point grid is needed to converge the $G_{0} W_{0}$ band gap and exciton energy to within $0.1 \mathrm{eV}$. We provide an extensive comparison with previous studies and explain agreement and variations in the results. It is demonstrated that too coarse $k$-point sampling and the interactions between the repeated layers have opposite effects on the band gap and exciton energy, leading to a fortuitous error cancellation in the previously published results.
\end{abstract}

DOI: 10.1103/PhysRevB.88.245309

PACS number(s): 73.22.-f, 78.20.Bh, 71.20.Nr, 71.35.-y

\section{INTRODUCTION}

Atomically thin two-dimensional (2D) materials such as graphene, hexagonal boron nitride, and transition-metal dichalcogenides (TMDC) possess unique electronic and optical properties including high intrinsic carrier mobilities, ${ }^{1-3}$ tunable band gaps, ${ }^{4,5}$ and strong light-matter interactions. ${ }^{6-9}$ These features, combined with the possibility of engineering their electronic properties further via strain, alloying, or stacking, make the 2D materials ideal as building blocks for new optoelectronic structures and devices with minimal sizes and performances surpassing present technologies.

After the intense focus on graphene, the TMDCs are now attracting increasing interest. ${ }^{10}$ This stems mainly from the greater variation in their electronic properties including both semiconducting and metallic behavior. So far, the most intensively studied single-layer TMDC is the semiconductor $\mathrm{MoS}_{2}$. Nanostructured forms of $\mathrm{MoS}_{2}$ have previously been explored as potential catalysts for desulferization of crude oil and more recently for (photo-) electrochemical hydrogen evolution. ${ }^{11-13}$ Bulk $\mathrm{MoS}_{2}$ is composed of two-dimensional sheets held together by weak van der Waals forces, and individual sheets can be isolated by exfoliation techniques similar to those used to produce graphene. ${ }^{1}$ Single layers of $\mathrm{MoS}_{2}$ therefore comprise highly interesting two-dimensional systems with a finite band gap and have recently been proposed for nanoelectronics applications. ${ }^{2}$

The optical properties of bulk $\mathrm{MoS}_{2}$ have been thoroughly studied experimentally. ${ }^{14-19}$ The absorption spectrum shows two distinct low-energy peaks at 1.88 and $2.06 \mathrm{eV}$, which are denoted by $A$ and $B$, respectively, ${ }^{20}$ and derive from direct transitions between a split valence band and the conduction band at the $\mathrm{K}$ point of the Brillouin zone. Their Rydberg satellites, Zeeman splitting, and dependence on crystal thickness have been investigated in detail. ${ }^{17}$ Recently, the quantum yield of luminescence from $\mathrm{MoS}_{2}$ was shown to increase dramatically when the sample thickness was changed from a few layers to a monolayer, ${ }^{7,8}$ indicating a transition to a direct band gap in the single layer.

In the past couple of years a number of theoretical studies of the electronic band structure and optical excitations in monolayer $\mathrm{MoS}_{2}$ have been published., ${ }^{41-26}$ These studies are based on many-body perturbation theory in the $G W$ approximation (mainly the non-self-consistent $G_{0} W_{0}$ approach) for the band structure and the Bethe-Salpeter equation (BSE) with a statically screened electron-hole interaction for the optical excitations. As is standard practice, the calculations have been performed on a supercell geometry where the $\mathrm{MoS}_{2}$ layers have been separated by $10-20 \AA$ vacuum and the Brillouin zone (BZ) was sampled on grids ranging from $6 \times 6$ to $15 \times 15$. With these parameters, $G_{0} W_{0}$ band gaps in the range $2.6-3.0 \mathrm{eV}$ and $G_{0} W_{0}$-BSE exciton binding energies of $0.6-1.1 \mathrm{eV}$ have been reported. Moreover, both direct ${ }^{21-25}$ and indirect ${ }^{4}$ band gaps have been found at the $G_{0} W_{0}$ level, while only direct gaps have been obtained with self-consistent $G W^{21}$ and $G W_{0} \cdot{ }^{4,26}$ When comparing these values, it should be kept in mind that both the size and nature of the band gap of $\mathrm{MoS}_{2}$ depend sensitively on the in-plane lattice parameter, $a{ }^{4}$

One of the most fundamental quantities describing the electronic structure of a material is the dielectric function. The dielectric properties of atomically thin 2D materials are quite different from their three-dimensional (3D) counterparts. ${ }^{27}$ For example, plasmons in 2D metals have acoustic dispersion relations $\left[\omega_{p}(q) \rightarrow 0\right.$ as $q \rightarrow 0$ ], and screening is generally much weaker, leading to strong exciton binding energies in 2D semiconductors. Reported static dielectric constants for monolayer $\mathrm{MoS}_{2}$ obtained using the supercell approach lie in the range 4.2-7.6 (for in-plane polarization). ${ }^{21,24,28}$ These values have been used to rationalize the exciton binding energy in $\mathrm{MoS}_{2}$ using the simple Mott-Wannier model.

In this paper, we present an in-depth study of the dielectric function, quasiparticle (QP) band structure, and excitonic states in monolayer $\mathrm{MoS}_{2}$. We focus on separating the spurious interlayer screening from the intrinsic intralayer screening in 
supercell geometries and on the consequences of the physics of 2D screening for the convergence of many-body excited-state calculations. The 3D macroscopic dielectric constant, as used for solids, converges to 1 for all $q$ vectors in the limit of infinite separation of the layers and is thus meaningless for a 2D material. We use an alternative approach to calculate the dielectric constant by averaging the total field over the material rather than the supercell. This 2D dielectric constant shows strong $q$ dependence for small wave vectors and becomes exactly 1 for $q=0$. This property has important consequences for the $k$-point convergence of many-body calculations.

In general, the use of a truncated Coulomb interaction is essential to avoid interlayer screening which decays slowly with the layer separation, $L$. The interlayer screening yields a too large dielectric constant for wave vectors $q<1 / L$. As a consequence, the $G_{0} W_{0}$ band gaps and exciton energies are $0.5 \mathrm{eV}$ too low on average for layer separations of around $20 \AA$. For larger layer separations, the strong $q$ dependence of the dielectric constant for small $q$ implies that a $k$-point grid of at least $45 \times 45$ is required to converge band gaps and exciton energies to $0.1 \mathrm{eV}$. For $k$-point grids below $15 \times 15$ the band gap is at least $0.5 \mathrm{eV}$ too large in the limit $L \rightarrow \infty$. Thus, the effects of interlayer screening and too coarse $k$-point grids partially cancel out, leading to reasonable values for the band gap and exciton binding energy with underconverged parameters as applied in previous studies.

The paper is organized as follows. In Sec. II we present $G_{0} W_{0}$ band structures and study the convergence of the gap with respect to interlayer separation and $k$-point sampling. In Sec. III we show calculations for the 2D dielectric constant and explain the origin of the slow $k$-point convergence of the band gap. In Sec. IV we present many-body calculations of the lowest excitons and analyze their convergence with layer separation and $k$-point sampling. Our conclusions are given in Sec. V.

\section{QUASIPARTICLE BAND STRUCTURE}

In this section we demonstrate that $G W$ band structures for monolayer $\mathrm{MoS}_{2}$ converge extremely slowly with respect to the interlayer separation. In order to obtain wellconverged results (within $0.1 \mathrm{eV}$ ), the use of a truncated Coulomb interaction is inevitable, along with a $k$-point grid of around $45 \times 45$. Previously reported calculations with the full Coulomb interaction have employed only separation between 10 and $20 \AA$ and used from $6 \times 6$ to $12 \times 12 k$ points. The resulting band structures are, however, somewhat saved by a fortunate error cancellation between the two effects.

\section{A. Computational details}

All our calculations have been performed with the projector augmented wave method code GPAw. ${ }^{29,30}$ The Kohn-Sham wave functions and energies of monolayer $\mathrm{MoS}_{2}$ were calculated in the local density approximation (LDA) using a plane-wave basis with cutoff energy $400 \mathrm{eV}$. The $4 s$ and $4 p$ semicore electrons of Mo were explicitly included in all calculations. Unless otherwise stated the calculations have been performed for the experimental lattice constant of $3.16 \AA$. One-shot $G_{0} W_{0}$ calculations were performed using the LDA wave functions and eigenvalues to obtain the $G_{0} W_{0} @$ LDA

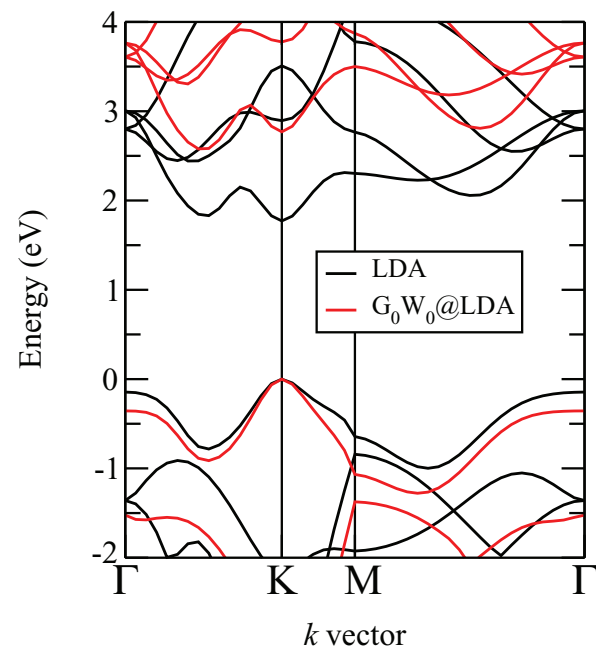

FIG. 1. (Color online) Band structure of monolayer $\mathrm{MoS}_{2}$ calculated with LDA and $G_{0} W_{0} @$ LDA using $45 \times 45 k$ points and a truncated Coulomb interaction to avoid interaction between periodically repeated layers. The valence-band tops have been aligned.

quasiparticle energies. A plane-wave cutoff of $50 \mathrm{eV}$ and 200 bands were used for the dielectric function, screened interaction, and $G W$ self-energy. Convergence with respect to these parameters has been checked very carefully. With these values, band gaps were found to be converged within around $10 \mathrm{meV}$. The plasmon pole approximation of Godby and Needs ${ }^{31}$ for the dielectric function was found to yield QP energies within $0.1 \mathrm{eV}$ of those obtained from full frequency dependence and was consequently used in all calculations. To avoid interaction between the periodically repeated $\mathrm{MoS}_{2}$ sheets, we have applied a truncated Coulomb interaction of the form $v_{c}(\mathbf{r})=(1 / r) \theta\left(R_{c}-z\right)$, following Refs. 32 and 33, in the calculation of both the dielectric function, $\epsilon$, and the screened potential, $W=\epsilon^{-1} v_{c}$. For details on the implementation of the $G W$ method in the GPAW code we refer to Ref. 34. We note that we have performed a numerical integration of the Coulomb interaction around each $q$ point in the Brillouin zone [see Eq. (9) in Sec. III C] when evaluating the head of the screened potential, $W_{\mathbf{0 0}}(\mathbf{q})$. This was found to be crucial for the $k$-point convergence both when employing the truncated and full Coulomb interaction.

\section{B. Results}

The band structure calculated using $45 \times 45 k$ points and the truncated Coulomb interaction is shown in Fig. 1. At the LDA level, we find a direct band gap at the K point of $1.77 \mathrm{eV}$, while the smallest indirect gap of $1.83 \mathrm{eV}$ occurs from $\Gamma$ to a point along the $\Gamma-\mathrm{K}$ direction. In contrast, $G_{0} W_{0}$ predicts an indirect gap of $2.58 \mathrm{eV}$ and a direct gap at $\mathrm{K}$ of $2.77 \mathrm{eV}$.

In Fig. 2 we show the convergence of both the direct and the indirect band gap with respect to the $k$-point grid for a fixed interlayer separation of $23 \AA$ (see Fig. 3 for the definition of $L)$. It is clear that a very dense $k$-point grid is needed in order to obtain well-converged results with the truncated Coulomb interaction. For $45 \times 45 k$ points, band gaps are converged within less than $0.1 \mathrm{eV}$, while this is already the case for $15 \times 15 k$ points with the bare Coulomb interaction. However, 


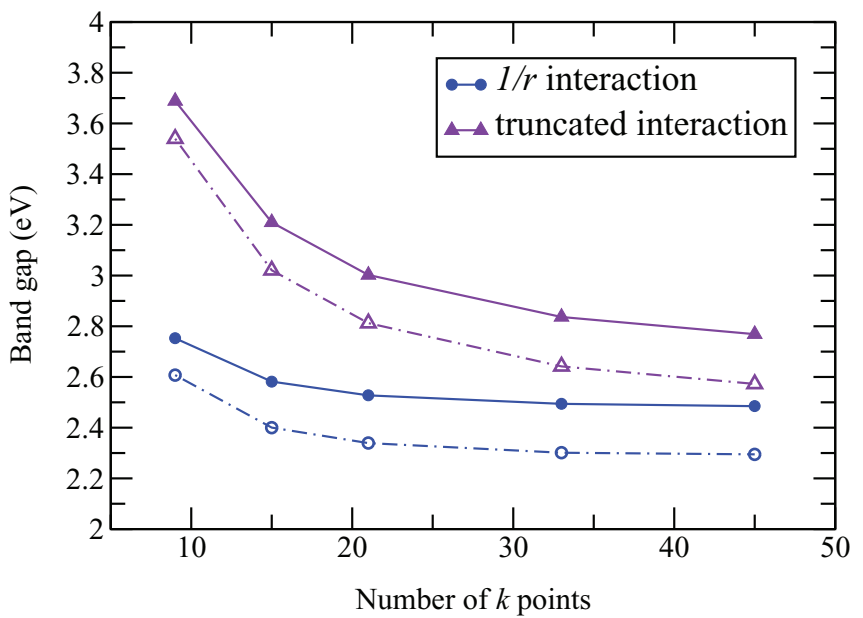

FIG. 2. (Color online) Direct (full symbols) and indirect (open symbols) $G_{0} W_{0}$ band gaps as function of the number of $k$ points in one of the in-plane directions for a layer separation of $L=23 \AA$.

the values obtained with the bare Coulomb interaction are too low as a result of interlayer screening. The slow convergence with respect to $k$ points when the truncation is used will be discussed in detail in Sec. III C.

We see that results do not converge independently with respect to the number of $k$ points and the interlayer separation. ${ }^{35}$ In Fig. 4, we plot the $L$ dependence of the direct band gap for different $k$-point samplings with the bare interaction. The $k$-point dependence becomes much stronger for large $L$. For $L \rightarrow \infty$, the values are expected to converge to the results calculated with the truncation (indicated by dotted lines). They seem to exhibit a linear $1 / L$ behavior only for $L>50 \AA$. Figure 5 shows all results and interpolated values in a contour plot as a function of $1 / L$ and the number of $k$ points. The effects of using more $k$ points and increasing $L$ are of different sign and partially cancel each other. This is the reason why different choices of the two parameters yield the same results. Especially, the band gaps calculated with $9 \times 9 k$ points and $L=23 \AA$ and $15 \times 15 k$ points and $L=43 \AA$ are the same as with $45 \times 45 k$ points and infinite $L$. This seems, however, coincidental, and we do not expect it to be the case for other systems.

We note that all calculations have been performed with a single $k$ point in the direction perpendicular to the layer. This is, however, insufficient for small interlayer distances.

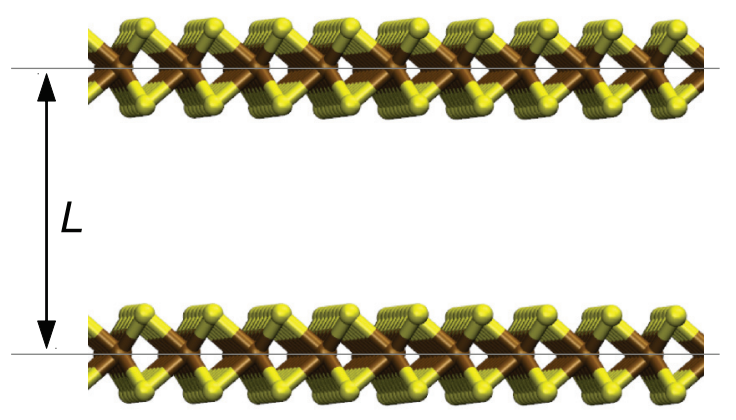

FIG. 3. (Color online) Definition of the interlayer separation, $L$.

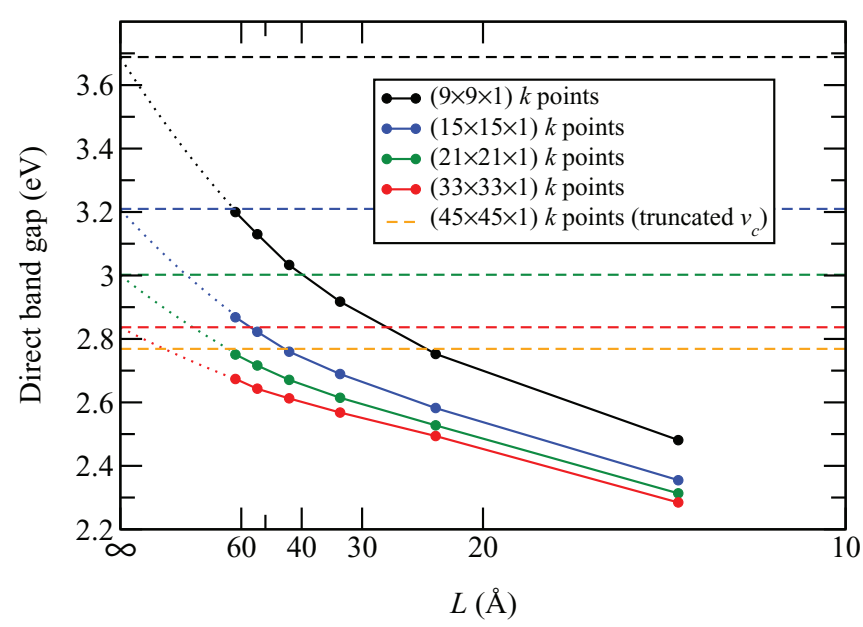

FIG. 4. (Color online) Direct $G_{0} W_{0}$ band gap plotted as a function of interlayer distance for different $k$-point samplings with the full $1 / r$ interaction. Dotted lines serve as a guide for the eye to extrapolate for $L \rightarrow \infty$. They were obtained by fitting all values for $L>30 \AA$, including the results with the Coulomb truncation, to a quadratic function. Dashed horizontal lines indicate the calculated values with the truncated Coulomb interaction.

For $L=13 \AA$, we find an increase of the band gap of around $0.2-0.3 \mathrm{eV}$ when at least $3 k$ points are used, for example. For $L>20 \AA$ or use of the truncation, this effect is negligible.

\section{Comparison with previous work}

In Table I we show our converged results obtained with the truncated Coulomb interaction and $45 \times 45 k$ points together with previous $G_{0} W_{0}$ results from the literature. For each reference we show the values used for the lattice constant, the interlayer separation, and the $k$-point sampling. It can be seen that all the previous calculations have used small layer separations and no truncation method. As pointed out in the

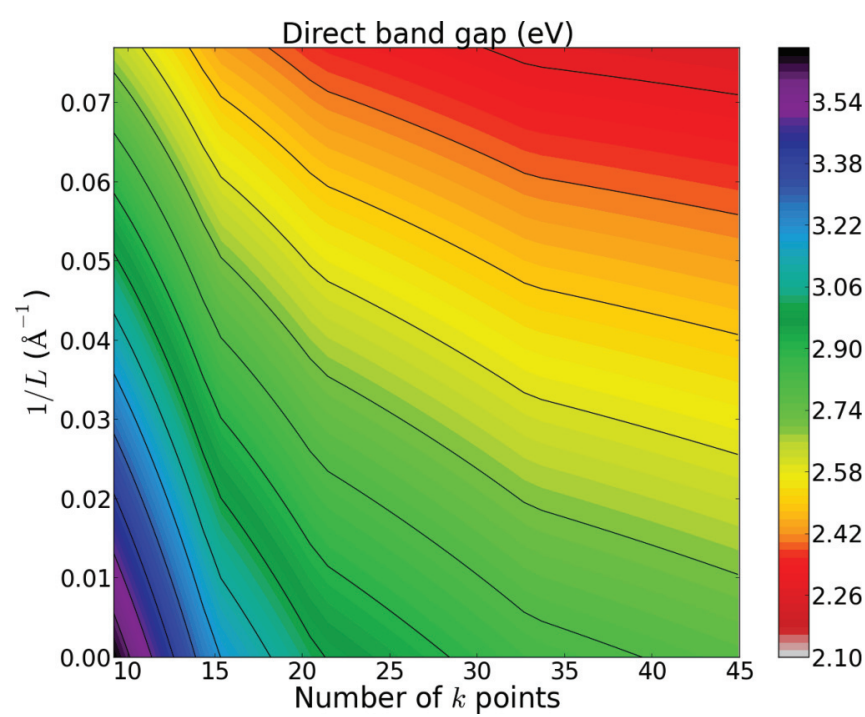

FIG. 5. (Color online) Contour plot of the direct $G_{0} W_{0}$ band gap as a function of the inverse interlayer distance and number of $k$ points in one of the in-plane directions with the full $1 / r$ interaction. Contour lines are separated by $0.1 \mathrm{eV}$. Interpolation from splines was used. 
TABLE I. Calculated $G_{0} W_{0}$ band gaps obtained in the present work and compared with previous results from the literature. All our calculations have been performed using a truncated Coulomb interaction.

\begin{tabular}{|c|c|c|c|c|c|c|}
\hline \multirow[b]{2}{*}{ Reference } & \multirow[b]{2}{*}{ Starting point } & \multirow[b]{2}{*}{$a(\AA)$} & \multirow[b]{2}{*}{ Number of $k$ points } & \multirow[b]{2}{*}{ Layer separation $(\AA)$} & \multicolumn{2}{|c|}{$E_{\text {gap }}(\mathrm{eV})$} \\
\hline & & & & & Direct & Indirect \\
\hline This work & LDA & 3.16 & $45 \times 45 \times 1$ & 23 (truncated $v_{c}$ ) & 2.77 & 2.58 \\
\hline This work & LDA & 3.19 & $45 \times 45 \times 1$ & 23 (truncated $v_{c}$ ) & 2.65 & 2.57 \\
\hline This work & LDA & 3.255 & $45 \times 45 \times 1$ & 23 (truncated $v_{c}$ ) & 2.41 & 2.51 \\
\hline Ref. 25 & LDA & 3.15 & $18 \times 18 \times 1$ & 24 & 2.41 & $\sim 2.40$ \\
\hline Ref. 4 & PBE & 3.16 & $12 \times 12 \times 1$ & 19 & $\sim 2.60$ & 2.49 \\
\hline Ref. 4 & PBE & 3.19 & $12 \times 12 \times 1$ & 19 & 2.50 & $\sim 2.55$ \\
\hline Ref. 4 & PBE & 3.255 & $12 \times 12 \times 1$ & 19 & 2.19 & 2.19 \\
\hline Ref. 21 & LDA & 3.16 & $8 \times 8 \times 2$ & 19 & 2.96 & - \\
\hline Ref. 22 & PBE & 3.18 & $12 \times 12 \times 1$ & $20+1 / L$ extrapolation & 2.97 & 3.26 \\
\hline Ref. 22 & PBE & 3.18 & $12 \times 12 \times 1$ & 20 & $\sim 2.60$ & $\sim 2.85$ \\
\hline Ref. 23 & LDA & 3.11 & $12 \times 12 \times 1$ & 13 & 2.57 & - \\
\hline Ref. 24 & HSE & 3.18 & $6 \times 6 \times 1$ & 15 & 2.82 & $\sim 3.00$ \\
\hline Ref. 36 & PBE & 3.19 & $15 \times 15 \times 1$ & 15 & 2.66 & - \\
\hline
\end{tabular}

preceding discussion, this gives a fast $k$-point convergence. A properly converged calculation, however, requires larger separations and thereby more $k$ points, but as a consequence of a cancellation of errors a calculation with $19 \AA$ of layer separation and $12 \times 12 k$ points yields almost the same band gaps as our converged result (within $0.15 \mathrm{eV}$ ). We are thus led to conclude that the reasonable agreement between our results and previous ones is to a large extent fortuitous.

Furthermore, the effect of strain can have a large impact on the $\mathrm{MoS}_{2}$ band gap. As demonstrated in Ref. 4, using $12 \times 12$ $k$ points and $19 \AA$ of layer separation, the $G_{0} W_{0}$ band gap for the experimental lattice constant of $3.160 \AA$ is indirect. With a lattice constant of $3.190 \AA$, corresponding to $1 \%$ strain, the gap changes to be direct. The lowering of the direct band gap becomes even more pronounced for larger lattice constants. As can be seen from the table, our converged results predict the same trend, in particular the decrease of the direct gap as a function of strain, with our values for the direct gap being generally $0.2 \mathrm{eV}$ larger. We note that for $3.255 \AA$ the smallest indirect transition occurs from the $\Gamma$ point at the valence band to the $\mathrm{K}$ point at the conduction band. This is also in agreement with Ref. 4 . In the partially self-consistent $G W_{0}$ calculations of Ref. 26, the opposite trend was found, namely, a transition from a direct to an indirect band gap for $\sim 5 \%$ strain. However, a layer separation of only $12 \AA$ and less than $9 \times 9 k$ points in the in-plane directions were used in that study.

In Ref. 22, the band gap was determined by extrapolating from $L=20 \AA$ to infinite layer separation, under the assumption that the gap scales linearly with the inverse distance between the layers. The obtained values for the direct and indirect band gaps are $\sim 3.0$ and $\sim 3.3 \mathrm{eV}$, respectively. This is consistent with our findings using the truncated Coulomb interaction, the same lattice constant of $3.18 \AA$, and the same (underconverged) $k$-point grid of $12 \times 12$ as in Ref. 22.

From our studies, we conclude that the $G_{0} W_{0} @$ LDA band gap of monolayer $\mathrm{MoS}_{2}$ is indirect with a value of $2.6 \mathrm{eV}$, while the direct gap is $2.8 \mathrm{eV}$, when the experimental lattice constant of $3.16 \AA$ is used. The question of how well the one-shot $G_{0} W_{0} @$ LDA approximation describes the true electronic structure of this system remains open. Partially self-consistent $G W_{0}{ }^{4}$ and fully self-consistent $G W^{21}$ calculations have been shown to consistently yield direct band gaps of 2.75-2.80 eV for a layer separation of $19 \AA$ and a $k$-point sampling of $12 \times 12 \times 1$ and $8 \times 8 \times 2$, respectively.

\section{STATIC SCREENING}

In this section we present a detailed investigation of the (static) dielectric properties of monolayer $\mathrm{MoS}_{2}$. This serves a dual purpose. First, it illustrates the origin of the slow convergence of the $G W$ results presented in the previous section (and the BSE results presented in the next section). Second, it shows that the usual definition of the macroscopic dielectric constant of a periodic solid is not meaningful when applied to a $2 \mathrm{D}$ system represented in a periodic supercell. We discuss the difference between 2D and 3D screening, which becomes particularly pronounced in the $q \rightarrow 0$ limit with large consequences for the calculation of optical excitations with static screening of the electron-hole interaction (see the next section).

\section{A. 3D macroscopic dielectric constant}

The microscopic dielectric function determines the relation between a weak external potential and the total potential in the material:

$$
V_{\mathrm{tot}}(\mathbf{r})=\int d \mathbf{r}^{\prime} \epsilon^{-1}\left(\mathbf{r}, \mathbf{r}^{\prime}\right) V_{\mathrm{ext}}\left(\mathbf{r}^{\prime}\right) .
$$

For a periodic system the dielectric function can be conveniently expressed in plane waves:

$$
\epsilon^{-1}\left(\mathbf{r}, \mathbf{r}^{\prime}\right)=\sum_{\mathbf{G} \mathbf{G}^{\prime}} \sum_{\mathbf{q}} e^{i(\mathbf{G}+\mathbf{q}) \mathbf{r}} \epsilon_{\mathbf{G G}^{\prime}}^{-1}(\mathbf{q}) e^{-i\left(\mathbf{G}^{\prime}+\mathbf{q}\right) \mathbf{r}^{\prime}},
$$

where $\mathbf{G}$ is a reciprocal lattice vector and $\mathbf{q}$ is a wave vector in the 1. BZ. Within the random-phase approximation (RPA) we have

$$
\epsilon_{\mathbf{G G}^{\prime}}(\mathbf{q}, \omega)=\delta_{\mathbf{G G}^{\prime}}-v_{c}(\mathbf{q}+\mathbf{G}) \chi_{\mathbf{G G}^{\prime}}^{0}(\mathbf{q}, \omega),
$$


where $\chi^{0}$ is the noninteracting density response function. Here, $v_{c}$ can be the Fourier representation of either the full or the truncated Coulomb interaction. For the calculations in this section we have used $50 \mathrm{eV}$ cutoff for the reciprocal-lattice vectors to account for local-field effects. The noninteracting response function, $\chi^{0}$, was constructed from LDA wave functions and energies including states up to $50 \mathrm{eV}$ above the Fermi level. All calculations were performed with the projector augmented wave method code GPAW. Details on the implementation of the dielectric function in the GPAW code can be found in Ref. 37.

It follows from Eq. (2) that the total potential resulting from a plane-wave external potential $V_{0} e^{i \mathbf{q} \cdot \mathbf{r}}$ has the form

$$
V_{\mathrm{tot}}(\mathbf{r})=\widetilde{V}_{\mathbf{q}}(\mathbf{r}) e^{i \mathbf{q} \cdot \mathbf{r}},
$$

where $\widetilde{V}_{\mathbf{q}}(\mathbf{r})$ is a lattice periodic function. We thus define the macroscopic dielectric constant as

$$
\frac{1}{\epsilon_{M}(\mathbf{q})} \equiv \frac{\left\langle\widetilde{V}_{\mathbf{q}}\right\rangle_{\Omega}}{V_{0}}=\epsilon_{\mathbf{0 0}}^{-1}(\mathbf{q}),
$$

where $\langle\ldots\rangle_{\Omega}$ denotes a spatial average over a unit cell. Note that in general $\epsilon_{M}(\mathbf{q}, \omega) \neq \epsilon_{\mathbf{0 0}}(\mathbf{q}, \omega)$ because of local-field effects. ${ }^{38,39}$

To explicitly demonstrate that Eq. (5) does not apply to lowdimensional materials, we have calculated the macroscopic dielectric constant as a function of the layer separation, $L$. The results are shown in Fig. 6 for different values of the in-plane momentum transfer $q$. We also show the dielectric constant corresponding to polarization orthogonal to the layer. Clearly, the macroscopic dielectric constant approaches unity for all $q$ vectors in the limit of large interlayer separation. This occurs because the total field is averaged over an increasingly larger vacuum region.

Previously reported values for the macroscopic dielectric constant of monolayer $\mathrm{MoS}_{2}$ lie in the range 4-8. ${ }^{21,24,28}$ In these calculations the $\mathrm{MoS}_{2}$ layers were separated by a $10-20-\AA$ vacuum. As can be seen from $\epsilon_{\|}(q=0)$ in Fig. 6,

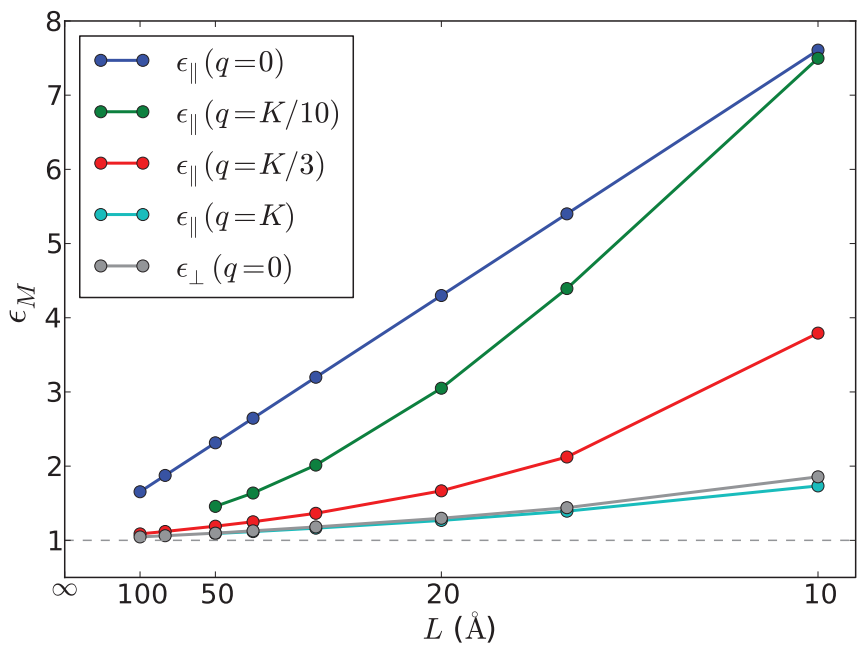

FIG. 6. (Color online) The 3D static macroscopic dielectric constant $1 / \epsilon_{\mathbf{0 0}}^{-1}(\mathbf{q})$ of monolayer $\mathrm{MoS}_{2}$ as a function of the interlayer separation, $L . \epsilon_{\|}$is the dielectric constant with polarization parallel to the monolayer, and $\epsilon_{\perp}$ is the dielectric constant for polarization orthogonal to the layer. this is consistent with our results. However, it should also be clear that numbers depend on the distance between layers and in fact are not meaningful.

\section{B. 2D macroscopic dielectric constant}

For a 2D material, the average of the total potential in the definition of the macroscopic dielectric constant must be confined to the region of the material. Since Eq. (4) still holds for a $2 \mathrm{D}$ material when $\mathbf{q}$ is confined to the plane of the material, we average the in-plane coordinates $\left(\mathbf{r}_{\|}\right)$ over the unit-cell area $A$ and the out-of-plane coordinate $(z)$ from $z_{0}-d / 2$ to $z_{0}+d / 2$, where $z_{0}$ denotes the center of the material and $d$ denotes its width. The 2D macroscopic dielectric constant then becomes

$$
\begin{aligned}
\frac{1}{\epsilon_{M}^{2 \mathrm{D}}\left(\mathbf{q}_{\|}\right)} & \equiv \frac{\left\langle\widetilde{V}_{\mathbf{q}}\right\rangle_{A, d}}{V_{0}} \\
& =\frac{2}{d} \sum_{G_{\perp}} e^{i G_{\perp} z_{0}} \frac{\sin \left(G_{\perp} d / 2\right)}{G_{\perp}} \epsilon_{\mathbf{G 0}}^{-1}\left(\mathbf{q}_{\|}\right),
\end{aligned}
$$

where the sum is over all $\mathbf{G}$ with $\mathbf{G}_{\|}=\mathbf{0}$. In this work we have taken $d=6.15 \AA$ corresponding to the interlayer separation in bulk $\mathrm{MoS}_{2}$. We shall return to the problem of choosing $d$ below.

The results for the static dielectric constant evaluated from Eq. (6) using the bare Coulomb interaction are shown in Fig. 7 for four different layer separations. The result for $L=d=6.15 \AA$ coincides with the $3 \mathrm{D}$ dielectric constant of bulk $\mathrm{MoS}_{2}$ given by Eq. (5). The result obtained with the truncated Coulomb interaction is shown in black; it represents the case of infinite layer separation. Before discussing the results, it is instructive to consider the potential arising from a

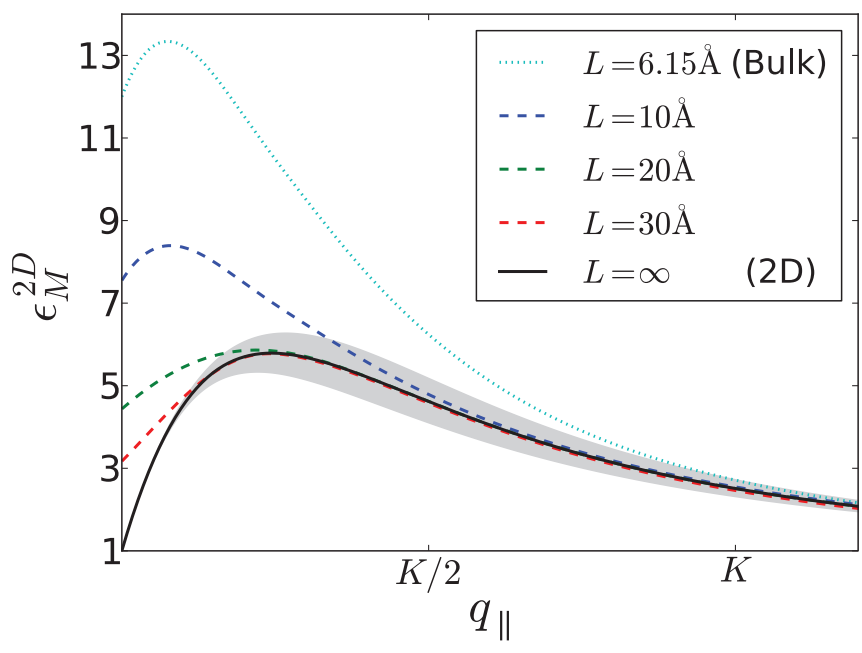

FIG. 7. (Color online) Static macroscopic dielectric constant for a single layer of $\mathrm{MoS}_{2}$ calculated along the $\Gamma-\mathrm{K}$ line. The calculations are performed using Eq. (6) with the microscopic dielectric constant, $\epsilon_{\mathbf{G G}^{\prime}}^{-1}(\mathbf{q})$, evaluated from Eq. (3) with either the bare Coulomb interaction (dotted and dashed lines) or truncated Coulomb interaction (full black line). The gray area represents the result obtained when the averaging region perpendicular to the layer, $d$, is varied by $\pm 10 \%$. The dotted line corresponds to a layer separation of $6.15 \AA$ and thus coincides with the dielectric constant of bulk $\mathrm{MoS}_{2}$. The curves have been interpolated from a $32 \times 32 q$-point mesh. 
2D charge-density fluctuation of the form

$$
n(\mathbf{r})=n_{0} e^{i \mathbf{q}_{\|} \cdot \mathbf{r}_{\|}} \delta(z) .
$$

The corresponding potential follows from Poisson's equation: ${ }^{40}$

$$
\phi(\mathbf{r})=\frac{n_{0}}{q_{\|}} e^{-i \mathbf{q}_{\|} \cdot \mathbf{r}_{\|}} e^{-q_{\|}|z|} .
$$

It follows that the potential perpendicular to the layer falls off exponentially over a characteristic distance of $1 / q_{\|}$. This explains why in general $\epsilon_{M}^{2 \mathrm{D}}\left(\mathbf{q}_{\|}\right)$coincides with the isolated layer result for $q_{\|} \gtrsim 1 / L$.

The variation of $\epsilon_{M}^{2 \mathrm{D}}$ when the parameter $d$ is changed by $\pm 10 \%$ is indicated by the shaded region in Fig. 7 . To the left of the maximum, $\epsilon_{M}^{2 \mathrm{D}}\left(\mathbf{q}_{\|}\right)$is insensitive to $d$ since the induced potential is more or less constant over the averaging region. To the right of the maximum, the variation in $\epsilon_{M}^{2 \mathrm{D}}\left(\mathbf{q}_{\|}\right)$follows the $\pm 10 \%$ variation in $d$. This is because for these wave vectors the induced potential has essentially vanished at the borders of the averaging region. In general, increasing (decreasing) $d$ will decrease (increase) $\epsilon_{M}^{2 \mathrm{D}}\left(\mathbf{q}_{\|}\right)$in the large wave-vector region.

Another characteristic feature of the potential in Eq. (8) is the $1 / q_{\|}$scaling, which should be compared with the $1 / q^{2}$ form of the Coulomb potential from a 3D charge oscillation. Since the noninteracting response function, $\chi_{\mathbf{0 0}}^{0}(\mathbf{q})$, scales as $\sim q^{2}$ for $q \rightarrow 0$ for both $2 \mathrm{D}$ and 3D systems, it follows from Eq. (3) that $\epsilon_{M}^{2 \mathrm{D}}(0)=1$, while this is in general not the case in three dimensions. In our calculations, the effect of interlayer interactions is eliminated by using a truncated Coulomb interaction of the form $v_{c}(\mathbf{r})=(1 / r) \theta\left(R_{c}-z\right)$. For $q_{z}=0$ and in the limit of small $q_{\|}$, the Fourier representation of the truncated Coulomb interaction becomes $v^{2 \mathrm{D}}(\mathbf{q})=\frac{4 \pi R_{c}}{|\mathbf{q}|}$; i.e., it scales as $1 / q$ as the potential from the $2 \mathrm{D}$ charge-density wave, ensuring the correct limit $\epsilon_{M}^{2 \mathrm{D}}(0)=1$.

Finally, we note that previous studies ${ }^{41,42}$ have employed a strict 2D model for the dielectric function in the small $q$ limit of the form $\epsilon\left(q_{\|}\right)=1+\alpha q_{\|}$. This form is convenient, as it leads to an analytical expression for the 2D screened interaction. ${ }^{27}$ Our definition differs by being a 3D (or quasi-2D) quantity valid for general $q_{\|}$.

\section{Screened interaction}

In Fig. 8 we show $\epsilon_{\mathbf{0 0}}^{-1}$ as a function of $q_{\|}$evaluated with and without the truncated Coulomb interaction. For small $q$, the two curves differ significantly due to the long-range nature of the induced potential Eq. (8). At large $q(\sim \mathrm{K} / 2)$, the induced potential decays within the cutoff range for the truncated Coulomb interaction, and therefore no difference can be seen between the two methods. We emphasize that neither of the dielectric constants shown in the figure can be interpreted as a dielectric constant of monolayer $\mathrm{MoS}_{2}$, since they give the average potential over the supercell and not over the $\mathrm{MoS}_{2}$ layer. In particular, their value will be highly dependent on the size of the unit cell (in the limit of infinite layer separation, both will equal 1 for all $q$ ). Nevertheless, this quantity is a crucial ingredient of both the $G W$ self-energy and the BSE kernel, as it provides the screening of the divergent term of the Coulomb interaction.

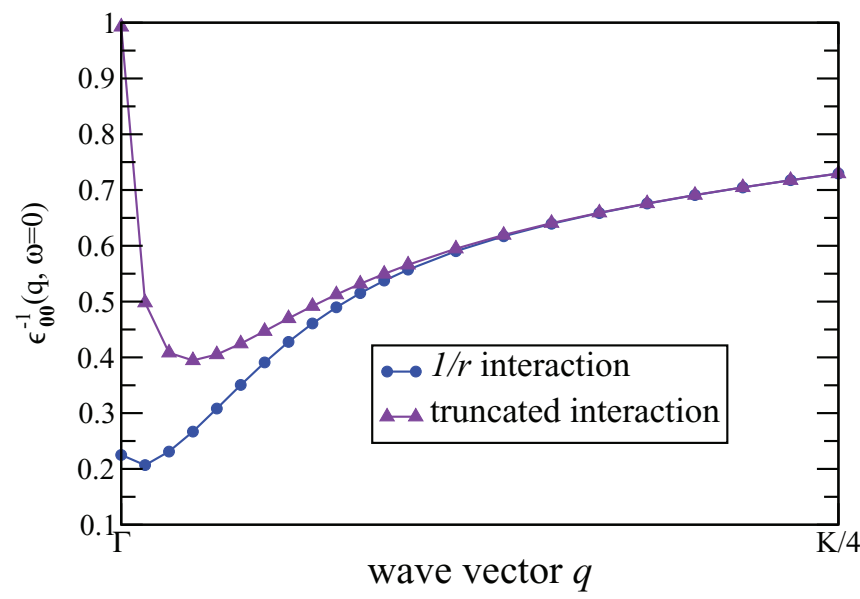

FIG. 8. (Color online) The 3D static inverse dielectric constant $\epsilon_{\mathbf{0 0}}^{-1}(\mathbf{q})$ of monolayer $\mathrm{MoS}_{2}$ calculated in the RPA for different values of in-plane momentum transfer $q$ along the $\Gamma$-K direction. The separation between layers is $L=20 \AA$. Note that neither of the quantities can be interpreted as the macroscopic dielectric constant of the monolayer (this quantity is the black curve in Fig. 7).

For $q=0$ the Coulomb kernel diverges and we approximate $W(q=0)$ by the integral

$$
\begin{aligned}
W_{\mathbf{0 0}}(\mathbf{q}=0) & =\frac{1}{\Omega_{\Gamma}} \int_{\Omega_{\Gamma}} d \mathbf{q} v_{c}(\mathbf{q}) \epsilon_{\mathbf{0 0}}^{-1}(\mathbf{q}) \\
& \approx \frac{1}{\Omega_{\Gamma}} \epsilon_{\mathbf{0 0}}^{-1}(\mathbf{q}=0) \int_{\Omega_{\Gamma}} d \mathbf{q} v_{c}(\mathbf{q}),
\end{aligned}
$$

where $\Omega_{\mathrm{BZ}}$ is the Brillouin-zone volume and $\Omega_{\Gamma}$ is a small volume containing $\mathbf{q}=0$. In isotropic systems, $\epsilon_{\mathbf{0 0}}^{-1}(\mathbf{q})$ is usually constant in the vicinity of $\mathbf{q}=0$ and the approximation works well. However, when $\epsilon^{-1}$ is evaluated with the truncated Coulomb interaction, $\epsilon_{\mathbf{0 0}}^{-1}$ acquires much more structure for small $q$ as can be seen from Fig. 8. Thus, for coarse $k$-point samplings we will underestimate the $\Gamma$-point screening since we simply use $\epsilon_{\mathbf{0 0}}^{-1}(\mathbf{q}=0)=1$.

The linear behavior of the screened interaction for small $q$ suggests that a better approximation for $W_{\mathbf{0 0}}(\mathbf{q}=0)$ would be

$$
W_{\mathbf{0 0}}(\mathbf{q}=0)=\frac{1}{\Omega_{\Gamma}} \int_{\Omega_{\Gamma}} d \mathbf{q} v_{c}(\mathbf{q})\left[1+\left.\mathbf{q} \cdot \nabla_{\mathbf{q}} \epsilon_{\mathbf{0 0}}^{-1}(\mathbf{q})\right|_{\mathbf{q}=0}\right] .
$$

Since the dielectric matrix in RPA is $\epsilon_{\mathbf{G G}^{\prime}}(\mathbf{q})=1-$ $v^{c}(\mathbf{q}) \chi_{\mathbf{G G}^{\prime}}^{0}(\mathbf{q})$, we can derive an analytic expression for the firstorder Taylor expansion in $q$ and its inverse. These quantities can be evaluated with vanishing additional cost, but we will leave the assessment of this approximation to future work.

\section{OPTICAL ABSORPTION SPECTRUM}

In this section we present many-body calculations of the optical absorption spectrum of monolayer $\mathrm{MoS}_{2}$ by solving the Bethe-Salpeter equation (BSE) under the standard assumption of static screening of the electron-hole interaction. As for the $G W$ band gap, we find that the use of a truncated Coulomb interaction is essential to avoid interlayer screening and obtain well-converged exciton binding energies. Furthermore, the very strong $q$ dependence of the $2 \mathrm{D}$ static dielectric function 
around $q=0$ leads to very slow $k$-point convergence for the exciton binding energy.

In order to obtain an accurate absorption spectrum including excitonic effects we calculate the response function from the BSE. Using the standard assumption of a static dielectric screening of the electron-hole (e-h) interaction, the $\mathrm{BSE}^{43}$ can be recast as an effective two-particle Hamiltonian, ${ }^{44}$ which is diagonalized in a basis of electron-hole pairs. In this way the excitonic eigenstates can be expressed as a linear combination of single-particle transitions:

$$
|\lambda\rangle=\sum_{v c k} A_{v c k}^{\lambda}|v c k\rangle,
$$

where $v, c$, and $k$ denote valence bands, conduction bands, and Brillouin-zone wave vectors, respectively. The absorption spectrum is proportional to the imaginary part of the macroscopic dielectric function, which in the Tamm-Dancoff approximation can be written

$$
\begin{aligned}
\epsilon_{2}(\omega)= & 2 \pi \lim _{\mathbf{q} \rightarrow 0} v_{c}(\mathbf{q}) \sum_{\lambda} \delta\left(\omega-E_{\lambda}\right) \\
& \times\left|\sum_{v c \mathbf{k}} A_{v c k}^{\lambda}\left\langle v \mathbf{k}-\mathbf{q}\left|e^{-i \mathbf{q} \cdot \mathbf{r}}\right| c \mathbf{k}\right\rangle\right|^{2},
\end{aligned}
$$

where $E_{\lambda}$ are the eigenvalues associated with $|\lambda\rangle$.

In all calculations we have included a single valence band and a single conduction band in the BSE Hamiltonian. We have tested that the first excitonic peak is completely unaffected if we instead include six valence bands and four conduction bands. This is also expected since the highest (lowest) valence (conduction) band is well isolated from the remaining bands at $\mathrm{K}$ where the exciton is centered (see Fig. 1). For the screening we have included 65 bands in the evaluation of the response function, which is sufficient for converged results. Increasing the number of bands to 300 affects the position of the first exciton by less than $10 \mathrm{meV}$. The plane-wave cutoff for the response function (local-field effects) was set to $50 \mathrm{eV}$, and we checked that the excitonic binding energy changed by less than $10 \mathrm{meV}$ when increasing the cutoff to $200 \mathrm{eV}$. The dependence on $k$-point sampling and interlayer separation will be examined below. Details on the implementation of the BSE method in the GPAW code can be found in Ref. 5 .

\section{A. Convergence tests}

In the lower panel of Fig. 9, we show the exciton binding energy as a function of interlayer separation calculated for different $k$-point samplings using the bare Coulomb interaction and the truncated Coulomb interaction. With the bare Coulomb interaction, the obtained results are far from convergence, even for $L=50 \AA$. The dependencies on the layer separation and number of $k$ points are very similar as for the quasiparticle gap discussed in Sec. II B, even on a quantitative level. Therefore, the optical gap, which is given by the difference of the QP gap and the exciton binding energy, is almost independent of $L$ and whether or not the truncation method is used, as shown in the upper panel. This is consistent with the observations in Ref. 22.

The convergence of the binding energy with respect to the $k$-point sampling is plotted in Fig. 10 for an interlayer

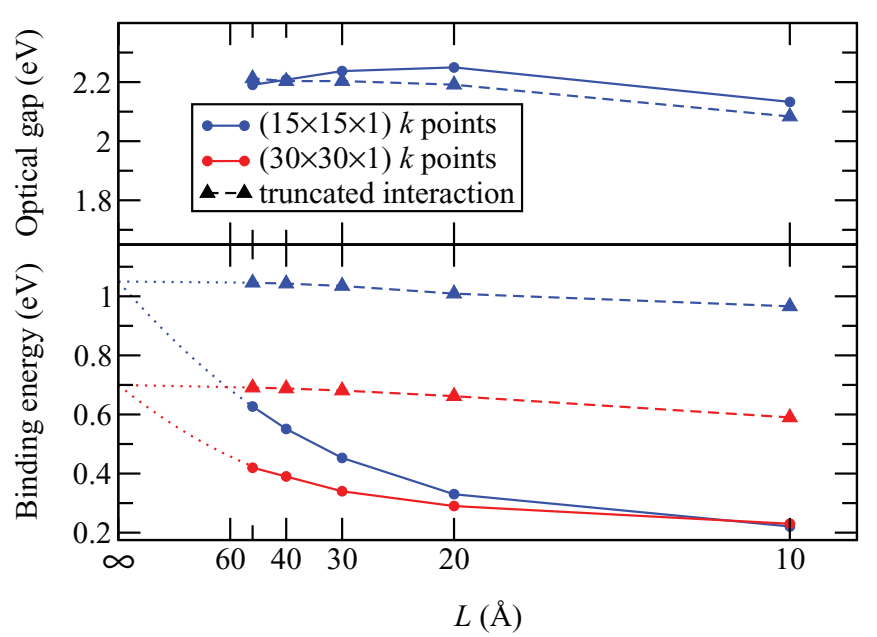

FIG. 9. (Color online) Optical gap and binding energy of the lowest exciton in monolayer $\mathrm{MoS}_{2}$ as a function of interlayer separation calculated from the BSE and the $G_{0} W_{0}$ quasiparticle gap. Results with the full $1 / r$ Coulomb interaction (full lines) and the truncated interaction (dashed lines) are shown. Dotted lines give an estimation for extrapolation to infinite $L$.

separation of $20 \AA$. The truncated Coulomb kernel gives a much slower convergence with respect to the number of $k$ points than the bare Coulomb interaction. However, it should be clear from Fig. 9 that the binding energy obtained with the bare Coulomb interaction converges to a value which is highly dependent on the interlayer separation. In Ref. 25, convergence was found with $18 \times 18 k$ points, but for a layer separation of only $24 \AA$. The obtained exciton binding energy was around $0.2 \mathrm{eV}$. According to our results, this is much too weak due to interlayer screening.

The slow $k$-point convergence observed when using the truncated Coulomb interaction is related to the $q$ dependence of the screening in two-dimensional systems. As demonstrated by Eq. (9) and Fig. 8 (purple curve), a too low $k$-point sampling leads to an underestimation of the screening in the vicinity

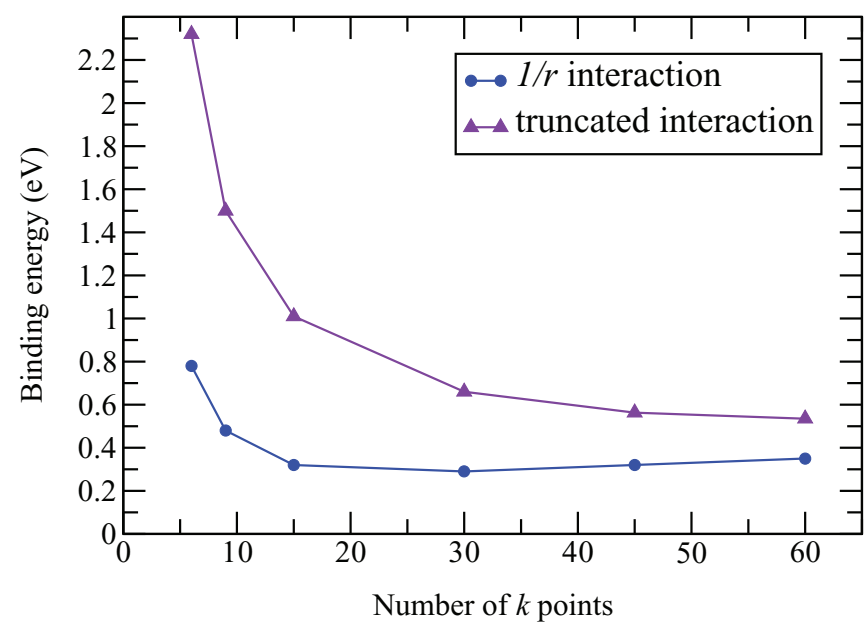

FIG. 10. (Color online) Binding energy of the lowest exciton in monolayer $\mathrm{MoS}_{2}$ as a function of $k$-point sampling for a supercell with a layer separation of $L=20 \AA$. 


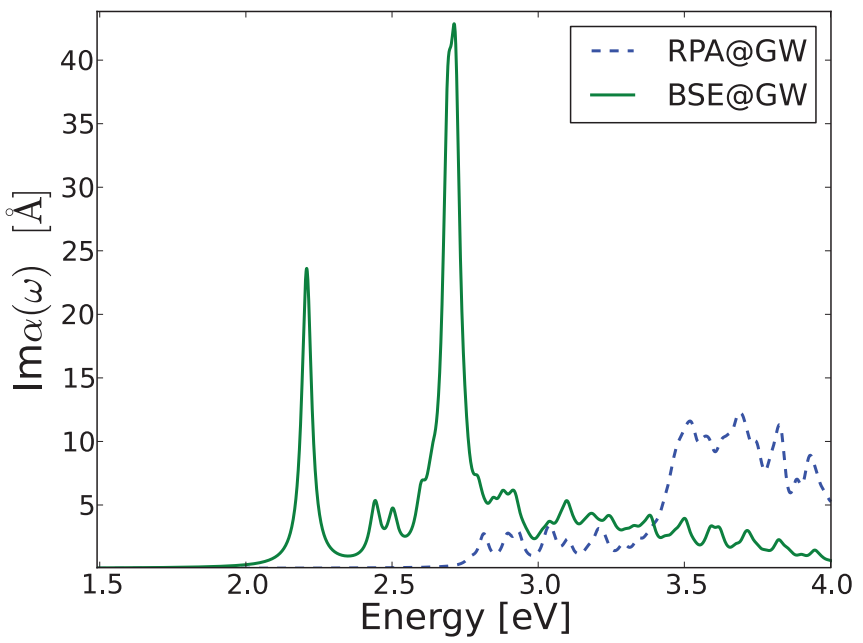

FIG. 11. (Color online) Absorption spectrum of single layer $\mathrm{MoS}_{2}$ calculated with the RPA and BSE using the $G_{0} W_{0}$ quasiparticle band structure. The calculation has been performed with a truncated Coulomb interaction to avoid interactions between repeated layers and with a $45 \times 45 k$-point grid.

of $q=0$ and thus an overestimation of the exciton binding energy.

We note that the slight increase in the binding energy with $k$ points obtained when using the full Coulomb interaction is not a result of decreased screening. Indeed the dielectric constant for this case is well converged already for a $k$-point sampling of $15 \times 15$ (see the slow wave vector dependence of the blue curve in Fig. 8). Instead, it is simply a result of the increased number of electron-hole basis states used to describe the exciton which lowers the BSE eigenvalue slightly (and thus increases the exciton binding energy). This effect is also present when using the truncated Coulomb interaction, but in this case the $k$-point variation in binding energy is dominated by the effect of increasing screening as discussed above (note that the screening effect is missed if $W$ is calculated on a coarse grid and extrapolated to a fine $k$-point grid before solving the BSE).

\section{B. Results}

From the convergence tests described above we conclude that the BSE calculations are (nearly) converged if we use a truncated Coulomb interaction and a $45 \times 45 k$-point sampling. With these settings we have calculated the BSE spectrum on top of a $G_{0} W_{0}$ quasiparticle band structure obtained with the same parameters. The resulting absorption spectrum is shown in Fig. 11. We also show an RPA calculation, i.e., neglecting electron-hole interactions in the BSE, performed on top of the same $G_{0} W_{0}$ band structure for comparison. With electron-hole interaction included, we obtain an exciton binding energy of $\sim 0.6 \mathrm{eV}$, whereas RPA does not show an exciton peak and simply gives an absorption edge at the band gap.

Experimentally, the absorption spectrum of single layer $\mathrm{MoS}_{2}$ exhibits a spin-orbit split peak around $1.9 \mathrm{eV}{ }^{7}$ Since we have not included spin-orbit coupling in our calculations, the spectrum in Fig. 11 only shows a single peak at low energies. However, it has previously been shown ${ }^{4,24}$ that the spin-orbit coupling does not have a large effect on the exciton binding energy and only results in a split excitonic peak. The main peak in the BSE@ $G_{0} W_{0}$ spectrum is situated at $2.2 \mathrm{eV}$, which is $0.3 \mathrm{eV}$ higher than the experimental value. At present we cannot say if this is due to an insufficient description of the quasiparticle gap within $G_{0} W_{0}$ or underestimation of the exciton binding energy in BSE with a static electron-hole interaction.

From the above discussion it should be clear that it is extremely challenging to converge the exciton binding energy with respect to interlayer separation and $k$ points. In general, the optical gap is much easier to converge with respect to interlayer separation than either the quasiparticle gap or the exciton binding energy. ${ }^{22,45}$ Nevertheless, for many physical applications it is of importance to obtain accurate values for both the quasiparticle gap and the exciton binding energy separately. In Ref. 22 the exciton binding energy was obtained by $1 / L$ extrapolation of the quasiparticle gap calculated in a range of interlayer separations between 10 and $20 \AA$ and assuming the same dependence for the exciton binding energy. Our results indicate that one should be cautious with such extrapolations. This is because the screening at different $q$ points has a very different dependence on interlayer separation, which results in different convergence behavior at different $k$-point samplings (see Fig. 9, full lines). The extrapolation procedure may therefore not give reliable results, since higher $k$-point samplings are required at larger interlayer separation. We are aware that the convergence issues may depend a lot on the implementation of the BSE method. However, we have previously performed the same calculations with the YAMBO $^{46}$ code, which produced very similar convergence behavior for quasiparticle gap and exciton binding energy (also using the truncated Coulomb cutoff and $45 \times 45 k$-point sampling).

\section{CONCLUSIONS}

We have presented a careful investigation of the dielectric properties, band gap, and excitonic states in a two-dimensional semiconductor exemplified by monolayer $\mathrm{MoS}_{2}$. We have demonstrated that the "standard" macroscopic dielectric constant used for solids is not applicable (meaningless) to supercells describing the 2D material as an infinite array of parallel sheets and therefore replaced it by a $2 \mathrm{D}$ version in which the induced field is averaged over the extent of the material rather than over the entire supercell. We showed that the effect of interlayer screening leads to underestimation of the band gap and exciton binding energy by up to more than $0.5 \mathrm{eV}$ for layer separations $<30 \AA$. The reason for this is that interlayer screening affects $\epsilon(q)$ for $q<1 / L$, where $L$ is the distance between layers in the supercell. Since it is the small $q$ limit of $\epsilon(q)$ that is most important for the screened interaction $W(q)=\epsilon^{-1}(q) / q^{2}$, the effect cannot be neglected. Here we have circumvented the problem by using a truncated Coulomb interaction that explicitly cuts off the interaction between neighboring layers.

The properly defined 2D dielectric function $\epsilon_{M}^{2 \mathrm{D}}(q)$ has a very sharp wave-vector dependence for small $q$ and satisfies $\epsilon_{M}^{2 \mathrm{D}}(0)=1$ in general. This has the consequence that quasiparticle and optical excitations obtained from the $G W$ and 
Bethe-Salpeter equation, respectively, require much denser $k$-point grids than experience from 3D systems would suggest. For $\mathrm{MoS}_{2}$ we find that a precision of $0.2 \mathrm{eV}$ requires $k$-point grids of at least $30 \times 30$. Interestingly, the effects of interlayer screening and too small $k$-point grids have opposite effects on the band gap and exciton energies, leading to fortuitous error cancellation. Our calculations applying the truncated Coulomb interaction and $45 \times 45 k$ points give $G_{0} W_{0} @$ LDA gaps of $2.77 \mathrm{eV}$ (direct) and $2.58 \mathrm{eV}$ (indirect) and a binding energy of the lowest exciton of $0.55 \mathrm{eV}$. This places the lowest exciton at $\sim 2.2 \mathrm{eV}$, which is $0.3 \mathrm{eV}$ higher than the experimental result.
This difference may be due to the $G_{0} W_{0} @$ LDA approximation or the use of static screening in the BSE.

\section{ACKNOWLEDGMENTS}

K.S.T. acknowledges support from the Danish Council for Independent Research Sapere Aude Program through Grant No. 11-1051390. The Center for Nanostructured Graphene is sponsored by the Danish National Research Foundation, Project No. DNRF58. *falco.hueser@fysik.dtu.dk

†thygesen@fysik.dtu.dk

${ }^{1}$ K. S. Novoselov, D. Jiang, F. Schedin, T. J. Booth, V. V. Khotkevich, S. V. Morozov, and A. K. Geim, Proc. Natl. Acad. Sci. USA 102, 10451 (2005).

${ }^{2}$ B. Radisavljevic, A. Radenovic, J. Brivio, V. Giacometti, and A. Kis, Nature Nanotechnology 6, 147 (2011).

${ }^{3}$ K. Kaasbjerg, K. S. Thygesen, and K. W. Jacobsen, Phys. Rev. B 85, 115317 (2012).

${ }^{4}$ H. Shi, H. Pan, Y.-W. Zhang, and B. I. Yakobson, Phys. Rev. B 87, 155304 (2013).

${ }^{5}$ J. Yan, K. W. Jacobsen, and K. S. Thygesen, Phys. Rev. B 86, 045208 (2012).

${ }^{6}$ L. Britnell et al., Science 340, 1311 (2013).

${ }^{7}$ K. F. Mak, C. Lee, J. Hone, J. Shan, and T. F. Heinz, Phys. Rev. Lett. 105, 136805 (2010).

${ }^{8}$ A. Splendiani, L. Sun, Y. Zhang, T. Li, J. Kim, C.-Y. Chim, G. Galli, and F. Wang, Nano Lett. 10, 1271 (2010).

${ }^{9}$ M. Bernardi, M. Palummo, and J. C. Grossman, Nano Lett. (unpublished).

${ }^{10}$ Q. H. Wang, K. Kalantar-Zadeh, A. Kis, J. N. Coleman, and M. S. Strano, Nature Nanotechnology 7, 699 (2012).

${ }^{11}$ M. V. Bollinger, J. V. Lauritsen, K. W. Jacobsen, J. K. Nørskov, S. Helveg, and F. Besenbacher, Phys. Rev. Lett. 87, 196803 (2001).

${ }^{12}$ T. F. Jaramillo, K. P. Jørgensen, J. Bonde, J. H. Nielsen, S. Horch, and I. Chorkendorff, Science 317, 100 (2007).

${ }^{13}$ X. Zong, H. Yan, G. Wu, G. Ma, F. Wen, L. Wang, and C. Li, J. Am. Chem. Soc. 130, 7176 (2008).

${ }^{14}$ R. F. Frindt and A. D. Yoffe, Proc. R. Soc. London A 273, 69 (1963).

${ }^{15}$ B. L. Evans and P. A. Young, Proc. R. Soc. London A 284, 402 (1965).

${ }^{16}$ J. A. Wilson and A. D. Yoffe, Adv. Phys. 18, 193 (1969).

${ }^{17}$ R. A. Neville and B. L. Evans, Phys. Status Solidi B 73, 597 (1976).

${ }^{18}$ G. L. Frey, S. Elani, M. Homyonfer, Y. Feldman, and R. Tenne, Phys. Rev. B 57, 6666 (1998).

${ }^{19}$ J. P. W. Newcomer and G. A. Samara, J. Appl. Phys. 81, 7934 (1997).

${ }^{20}$ R. Coehoorn, C. Haas, and R. A. de Groot, Phys. Rev. B 35, 6203 (1987).

${ }^{21}$ T. Cheiwchanchamnangij and W. R. L. Lambrecht, Phys. Rev. B 85, 205302 (2012).
${ }^{22}$ H.-P. Komsa and A. V. Krasheninnikov, Phys. Rev. B 86, 241201 (2012).

${ }^{23}$ C. Ataca and S. Ciraci, J. Phys. Chem. C 115, 13303 (2011).

${ }^{24}$ A. Ramasubramaniam, Phys. Rev. B 86, 115409 (2012).

${ }^{25}$ A. Molina-Sánchez, D. Sangalli, K. Hummer, A. Marini, and L. Wirtz, Phys. Rev. B 88, 045412 (2013).

${ }^{26}$ H. J. Conley, B. Wang, J. I. Ziegler, R. F. Haglund, S. T. Pantelides, and K. I. Bolotin, Nano Lett. 13, 3626 (2013).

${ }^{27}$ L. V. Keldysh, JETP Lett. 29, 658 (1979).

${ }^{28}$ A. Molina-Sanchez and L. Wirtz, Phys. Rev. B 84, 155413 (2011).

${ }^{29}$ J. Enkovaara et al., J. Phys.: Condens. Matter 22, 253202 (2010).

${ }^{30}$ The GPAW code is available as a part of the CAMPOS software: www.camd.dtu.dk/software.

${ }^{31}$ R. W. Godby and R. J. Needs, Phys. Rev. Lett. 62, 1169 (1989).

${ }^{32}$ C. A. Rozzi, D. Varsano, A. Marini, E. K. U. Gross, and A. Rubio, Phys. Rev. B 73, 205119 (2006).

${ }^{33}$ S. Ismail-Beigi, Phys. Rev. B 73, 233103 (2006).

${ }^{34}$ F. Hüser, T. Olsen, and K. S. Thygesen, Phys. Rev. B 87, 235132 (2013).

${ }^{35}$ C. Freysoldt, P. Eggert, P. Rinke, A. Schindlmayr, and M. Scheffler, Phys. Rev. B 77, 235428 (2008).

${ }^{36}$ Y. Ding, Y. Wang, J. Ni, L. Shi, S. Shi, and W. Tang, Physica B 406, 2254 (2011).

${ }^{37}$ J. Yan, J. J. Mortensen, K. W. Jacobsen, and K. S. Thygesen, Phys. Rev. B 83, 245122 (2011).

${ }^{38}$ S. Adler, Phys. Rev. 126, 413 (1962).

${ }^{39}$ N. Wiser, Phys. Rev. 129, 62 (1963).

${ }^{40}$ This is most easily seen by performing a 3D Fourier transformation of $\delta n$, then multiplying by $1 / q^{2}$, and Fourier transforming back to real space.

${ }^{41}$ P. Cudazzo, I. V. Tokatly, and A. Rubio, Phys. Rev. B 84, 085406 (2011).

${ }^{42}$ T. C. Berkelbach, M. S. Hybertsen, and D. R. Reichman, Phys. Rev. B 88, 045318 (2013).

${ }^{43}$ E. E. Salpeter and H. A. Bethe, Phys. Rev. 84, 1232 (1951).

${ }^{44}$ G. Strinati, Phys. Rev. B 29, 5718 (1984).

${ }^{45}$ L. Wirtz, A. Marini, and A. Rubio, Phys. Rev. Lett. 96, 126104 (2006).

${ }^{46}$ A. Marini, C. Hogan, M. Grüning, and D. Varsano, Comput. Phys. Commun. 180, 1392 (2009). 